\title{
Mediating Effects of Cognitive Effort and Depression on Intelligence, Memory, and Executive Functions in Individuals with Mild Traumatic Brain Injury
}

\author{
Kyoung-Sae $\mathrm{Na}^{1}$, Han-Yong Jung ${ }^{2}$, Soyoung Irene Lee ${ }^{2}$, and Shin-Gyeom Kim² \\ 'Department of Psychiatry, Gachon University Gil Medical Center, Incheon, Republic of Korea \\ ${ }^{2}$ Department of Psychiatry, Soonchunhyang University Bucheon Hospital, Bucheon, Republic of Korea
}

\begin{abstract}
Objective Mild traumatic brain injury (mTBI) is frequently associated with psychiatric symptoms and cognitive dysfunction, as well as with the receipt of workers' compensation, as many mTBIs occur due to work-related accidents. We hypothesized that depression and insufficient cognitive effort mediate the relationship between sociodemographic variables and cognitive dysfunction in mTBI.

Methods A retrospective chart review study was conducted using 115 records of patients with mTBI. Cognitive effort was measured based on scores on the Rey 15-Item Test. Multivariate linear regression analysis was performed to examine factors predictive of cognitive functions. Path analysis was subsequently performed to investigate the mediating effects of depression and cognitive effort in relation to receipt of workers' compensation and demographic variables.

Results Fifteen of the 115 participants (13.0\%) received failing scores on the Rey 15-Item Test, which indicated insufficient cognitive effort. Path analysis indicated that cognitive effort mediated the effects of age and workers' compensation on cognitive functions.

Conclusion Given the significant mediating effects of cognitive effort on cognitive performance, it is important to address patient motivation and encourage mTBI patients covered by workers' compensation to perform tests with authentic effort.
\end{abstract}

Psychiatry Investig 2014;11(2):112-118

Key Words Neuropsychological test, Brain concussion, Depression, Mental process, Compensation.

\section{INTRODUCTION}

Traumatic brain injury (TBI) is a common condition associated with substantial psychosocial and occupational impairment. According to the Centers for Disease Control and Prevention (CDC), 1.7 million people sustain a TBI in the United States annually. ${ }^{1}$ Although the mechanism by which trauma induces neuropsychiatric symptoms is still under investigation, the most widely accepted explanation is that accelerating and decelerating physical forces result in diffuse axonal injury by shearing the brain parenchyma within the skull. ${ }^{2-4}$

Mild TBI (mTBI) refers to a brief change in mental status or

Received: March 4, 2013 Revised: July 26, 2013

Accepted: September 7, 2013 Available online: April 11, 2014

$\triangle$ Correspondence: Han-Yong Jung, MD, PhD

Department of Psychiatry, Soonchunhyang University Bucheon Hospital, 170 Jomaru-ro, Wonmi-gu, Bucheon 420-767, Republic of Korea

Tel: +82-32-621-5232, Fax: +82-32-621-6950, E-mail: hanyjung@schmc.ac.kr

(a) This is an Open Access article distributed under the terms of the Creative Commons Attribution Non-Commercial License (http://creativecommons.org/licenses/by$\mathrm{nc} / 3.0$ ) which permits unrestricted non-commercial use, distribution, and reproduction in any medium, provided the original work is properly cited. consciousness. ${ }^{1}$ It is estimated that $70-90 \%$ of TBI patients are classified as having mTBI. ${ }^{5}$ The term "mild" in mTBI implies minimal causative neuronal injury with the expectation of temporary mild symptoms and spontaneous recovery. However, mTBI can be a serious condition that encompasses various neuropsychiatric symptoms, such as amnesia, inattention, executive dysfunction, headache, dizziness, and depression. ${ }^{6}$ Additionally, although most patients with mTBI completely recover within 1-3 months, ${ }^{7}$ some continue to experience sustained neuropsychiatric symptoms more than a year after injury. ${ }^{8} \mathrm{Sev}$ eral studies have suggested that motivation and secondary gain significantly influence cognitive performance in mTBI. ${ }^{9} \mathrm{~Pa}-$ tients with mTBI also tend to complain of more subjective symptoms than do those with moderate or severe TBI. ${ }^{8,10}$ Subjective and cognitive symptoms are more predictive of selfperceived symptoms 1 year post-concussion than is injury severity or sociodemographic factors. ${ }^{8}$ Additionally, depression substantially disturbs post-concussion symptoms in mTBI. ${ }^{11}$ Taken together, this evidence supports the importance of the roles of psychiatric symptoms, income source (workers' com- 
pensation or other), and cognitive effort on cognitive outcomes in mTBI.

However, it remains uncertain which variables are major determinants of outcomes related to cognitive functions. It is known that MTBI is associated with receipt of workers' compensation and that receipt of workers' compensation seems to influence cognitive effort. It is also plausible that depression may decrease motivation, decreasing cognitive effort. Therefore, it is important to investigate the mediating effects of these variables on overall cognitive functioning. In this study, we conducted path analysis to examine the potential mediating effects of depression, compensation, and cognitive effort on the relationship between sociodemographic factors and cognitive functions, including intelligence, memory, and executive functions.

\section{METHODS}

\section{Subjects}

This retrospective chart review study was conducted using patient records from March 2001 to March 2010. We assessed computerized sociodemographic and neuropsychological records from the Traumatic Brain Injury Center at the University Hospital. Patients with mTBI were included in the study based on medical records, and mTBI was defined, following the definition used by the $\mathrm{CDC},{ }^{12}$ as a head injury resulting from non-penetrating trauma and/or swaying (accelerating and decelerating) forces with one or more of the following conditions attributable to the head injury during the surveillance period: 1) Any period of observed or self-reported transient confusion, disorientation, or impaired consciousness; 2) Any period of observed or self-reported dysfunction of memory (amnesia) around the time of injury; 3) Observed signs of other neurological or neuropsychological dysfunction, such as seizures acutely following head injury; or 4) Headache, dizziness, irritability, fatigue, or poor concentration identified soon after the injury with evidence of loss of consciousness or altered consciousness. The exclusion criteria suggested by the CDC were: 1) Loss of consciousness lasting longer than 30 minutes; 2) Post-traumatic amnesia lasting longer than 24 hours; or 3) Penetrating craniocerebral injury. This study protocol was approved by the local Institutional Review Board (IRB) at Soonchunhyang University Bucheon Hospital.

\section{Instruments}

\section{Cognitive effort: Rey's 15-Item Test}

Rey's 15-Item Test was initially developed to detect malingering with respect to memory function. ${ }^{13}$ During the test, patients were instructed to look at five rows of three characters each, totaling 15 different items. After 10 seconds, the cards were withdrawn, and patients were instructed to recall and draw the 15 items. The score was defined as the number of items drawn by a patient. Because this test is easy to use and not time consuming, it is one of the most frequently used by neuropsychological experts in cases of workers' compensation and injury-related litigation. ${ }^{14}$ There is reasonable consensus that the cut-off point for insufficient cognitive effort is a score of eight or less. ${ }^{15}$

\section{Intelligence: Korean Wechsler Adult Intelligence Scale-Revised (K-WAIS-R)}

The K-WAIS-R is a standardized version of the Wechsler Adult Intelligence Scale translated into Korean. ${ }^{16}$ It consists of six verbal subtests (information, digit span, vocabulary, arithmetic, comprehension, and similarities) and five performance subtests (picture completion, picture arrangement, block design, digit symbol, and object assembly). The Intelligence Quotient (IQ) portion of the K-WAIS-R was standardized in a normal population to a mean of 100 with a standard deviation (SD) of 15. IQ scores were used in the current study to represent the intelligence domain of cognitive functioning in the path analysis.

\section{Memory: Rey-Kim memory test}

The Rey-Kim memory test evaluates both auditory and visual memory.

\section{Auditory memory: Rey-Kim Auditory Verbal Learning Test}

The Rey-Kim Auditory Verbal Learning Test (K-AVLT) is a modified version of the Rey Auditory Verbal Learning Test that was translated into Korean. ${ }^{17}$ This test consists of three steps. First, subjects listen to 15 consecutive words, followed by a free recall of the 15 words. Five of these trials are performed with the same 15 words. Second, subjects are instructed to again recall the 15 words 20 minutes later (delayed recall). Third, immediately after the delayed recall, 50 words are presented to patients; 15 of these are the same as those in the earlier trials, and subjects are instructed to select these 15 words (delayed recognition). Scores are calculated based on the total number of recalled words at each trial, delayed recall, and delayed recognition.

\section{Visual memory: Rey-Kim Complex Figure Test}

The Rey-Kim Complex Figure Test (K-CFT) is a standardized Korean version of the Rey-Osterrieth Complex Figure Test (ROCFT). ${ }^{17}$ It comprises three steps. First, patients are instructed to copy a complex figure (drawing). Second, patients are directed to recall and draw the complex figure (immediate recall). Third, 20 minutes after the second step, patients are instructed to draw the complex figure again (delayed recall). 
Scores are calculated based on the form and parts of the drawn complex figure.

Like IQ, the Rey-Kim memory test provides a standardized score, the memory quotient (MQ), which was used to represent the final dependent variable in the path model.

\section{Executive Functions: Executive Intelligence Test}

\section{Inhibition: Stroop test}

The Stroop test consists of three trials: a word trial, a color trial, and a word-color interference trial. ${ }^{18}$ This study used the translated Korean version. ${ }^{19}$ In the word-naming trial, the participants were told to read aloud four color words printed in black. In the color-naming trial, the words were printed in the color to which they referred (i.e., word and color were congruent), and the participants were told to read the words aloud. Finally, in the color-word interference trial, the words were incongruent with their ink color, and the participants were instructed to say the name of the ink color and not read the word. In this study, the results were presented in terms of response times (i.e., the time between the presentation of the target stimulus and the response).

\section{Verbal fluency: Controlled Oral Word Association Test}

The Controlled Oral Word Association (COWAT) Test is a standardized version of a word fluency test. ${ }^{20}$ This test measures phonemic and semantic fluencies. In the phonemic fluency test, participants are told to list as many words as they can think of that begin with the three Korean letters that resemble the letters "g," "o," and "s" during three separate 1-minute trials. In the semantic fluency test, participants are instructed to produce exemplars of fruit and animal categories during two separate 1-minute trials. In this study, the sum of the phonemic and semantic fluency scores was used to indicate verbal fluency.

\section{Design fluency: design fluency test}

The design fluency test used was a Korean standardized version of the Ruff Figural Fluency Test. ${ }^{21}$ Patients were instructed to connect five separate points in as many ways as possible during 1 minute. Total scores were calculated based on the number of different designs made in three trials.

The standardized score for this test, the executive intelligence quotient (EIQ), along with the IQ and MQ, was used in the path model in this study.

\section{Subjective psychiatric symptoms: Symptom Checklist 90-Revised Version}

The Symptom Checklist 90-Revised (SCL-90-R) is a 90-item, self-administered, multi-dimensional inventory designed to measure the current level of symptoms experienced by an individual within the last 7 days. ${ }^{22,23}$ The SCL-90-R was validated for a broad range of populations, including healthy people and those suffering with mTBI. ${ }^{24,25}$ Among the nine dimensions in the SCL-90-R, we used the depression subscale (13 items) in this study.

\section{Statistical analysis}

Demographic and clinical data were compared to determine group differences after participants were divided into two groups: those who failed (scores of 8 or lower, indicating low levels of cognitive effort) and those who passed (scores of 9 or higher, indicating high levels of cognitive effort) the Rey 15Item Test. Dichotomous variables were analyzed with chi-square tests, and continuous variables were analyzed using independent t-tests or Mann-Whitney tests, according to the normality of their distributions as assessed by the Shapiro-Wilk test.

Multivariate linear regression analysis was performed to reveal the factors predictive of cognitive functions. Age, gender, education, receipt of workers' compensation, Rey 15-Item Test scores, imaging abnormalities (hemorrhagic contusion), and SCL-90-R depression scores were included.

Path analysis was subsequently conducted to examine the mediating effects of depression and cognitive effort on cognitive functions. As depression can lower motivation, we set the SCL-90-R depression scores as the first mediating factor that might influence cognitive effort. All statistical analyses were conducted using Stata 12.1 (StataCorp LP, TX, USA).

\section{RESULTS}

\section{Subjects and cognitive effort}

A total of 115 patients were included. Demographic data are presented in Table 1. The Rey 15-Item Test mean (SD) score was 11.71 (2.87). Fifteen of 115 patients (13.0\%) scored 8 or less, and were thus classified in the failure (low cognitive effort) group; only 36 (31.3\%) patients had perfect scores.

\section{The relationship between cognitive effort and psychiatric symptoms and cognitive functioning}

Major cognitive functions, including intelligence, memory, and executive functions, and depression were analyzed according to whether subjects had passed or failed Rey's 15-Item Test (Table 2). The group that failed (low cognitive effort) had significantly lower scores on most of the cognitive tests addressing intelligence, memory, and executive functions as compared with the group that passed (high cognitive effort). There were no significant differences in depression severity between the two groups, and no significant differences in cognitive and psychiatric symptoms between patients with and without imaging 
Table 1. Demographic and clinical data of patients with mild traumatic brain injury $(\mathrm{N}=115)$

\begin{tabular}{lc}
\hline Variable & Mean (SD) or number (\%) \\
\hline Age & $40.8(12.5)$ \\
Gender (male) & $89(77.4)$ \\
Education (year) & $10.9(3.4)$ \\
Time since injury (month) & $10.6(7.2)$ \\
Compensation & \\
$\quad$ Workers' compensation & $35(30.4)$ \\
\multicolumn{1}{c}{ Others } & $80(69.6)$ \\
Abnormalities in MRI & $52(45.2)$ \\
Rey 15-item test scores & $11.7(2.9)$ \\
\hline
\end{tabular}

All data were presented as mean (SD) or number (\%). MRI: magnetic resonance imaging

Table 2. Comparison of cognitive function and psychiatric symptoms between pass and failure groups in Rey 15-Item Test

\begin{tabular}{|c|c|c|c|}
\hline \multirow{3}{*}{ Variable } & \multicolumn{2}{|c|}{ Rey 15-Item Test } & \multirow{3}{*}{$\mathrm{p}$ value } \\
\hline & Failure $(\mathrm{N}=15)$ & Pass $(\mathrm{N}=100)$ & \\
\hline & Mean (SD) & Mean (SD) & \\
\hline \multicolumn{4}{|l|}{ Intelligence } \\
\hline IQ & $86.8(10.2)$ & $95.0(12.5)$ & 0.02 \\
\hline VIQ & $90.6(9.9)$ & $96.4(12.7)$ & 0.10 \\
\hline PIQ & $82.9(11.1)$ & $93.6(13.0)$ & $<0.01$ \\
\hline EIQ & $70.7(17.7)$ & $85.2(19.4)$ & 0.01 \\
\hline MQ & $80.3(18.9)$ & $95.5(14.9)$ & $<0.01$ \\
\hline SCL-90-R depression & $69.3(18.6)$ & $62.7(13.0)$ & 0.26 \\
\hline
\end{tabular}

IQ: intelligence quotient, VIQ: verbal intelligence quotient, PIQ: performance intelligence quotient, EIQ: executive intelligence quotient, MQ: memory quotient, SCL-90-R: Symptom Checklist 90-Revised version

abnormalities were observed (data not shown).

\section{Multivariate linear regression analysis}

Separate multivariate linear regression analyses were conducted for intelligence, memory, and executive functions (Table 3). Cognitive effort was a common risk factor for all three cognitive components. Depression had an inverse influence on intelligence but not on memory or executive functions. Age had a positive influence on intelligence and memory.

\section{Path model for the mediating effects of cognitive effort and depression on cognitive performance}

The path model showed satisfactory results for goodness-offit: root mean squared error of approximation (RMSEA) $=0.002$, comparative fit index $(\mathrm{CFI})=1.000$, Tucker-Lewis index $(\mathrm{TLI})=1.000$, and standardized root mean squared residual $(\mathrm{SRMR})=0.056$. The direct and indirect effects of demographic and clinical variables with a standardized coefficient $\beta$ are presented in Figure 1. Age had both direct and indirect effects on
Table 3. Multiple linear regression analysis for intelligence, memory, and executive function

\begin{tabular}{|c|c|c|c|c|c|c|}
\hline \multirow{2}{*}{ Variable } & \multicolumn{2}{|c|}{ IQ } & \multicolumn{2}{|c|}{ MQ } & \multicolumn{2}{|c|}{ EIQ } \\
\hline & $\beta$ & $\mathrm{p}$ & $\beta$ & $\mathrm{p}$ & $\beta$ & $\mathrm{p}$ \\
\hline Age & 0.44 & $<0.01$ & 0.42 & $<0.01$ & 0.19 & 0.12 \\
\hline Gender & 0.03 & 0.79 & -0.15 & 0.13 & -0.16 & 0.11 \\
\hline Education (year) & -0.06 & 0.58 & -0.10 & 0.33 & -0.05 & 0.62 \\
\hline Compensation & -0.02 & 0.81 & 0.00 & 0.97 & -0.09 & 0.41 \\
\hline Imaging & -0.05 & 0.61 & 0.07 & 0.50 & 0.15 & 0.14 \\
\hline Rey 15-Item & 0.49 & $<0.01$ & 0.53 & $<0.01$ & 0.45 & $<0.01$ \\
\hline SCL-90-R depression & -0.23 & 0.05 & -0.11 & 0.34 & -0.08 & 0.48 \\
\hline
\end{tabular}

IQ: intelligence quotient, MQ: memory quotient, EIQ: executive intelligence quotient, SCL-90-R: Symptom Checklist 90-Revised version. Reference values: gender $=$ female, compensation $=$ nonworkers' compensation, imaging=non-abnormal findings on magnetic resonance imaging

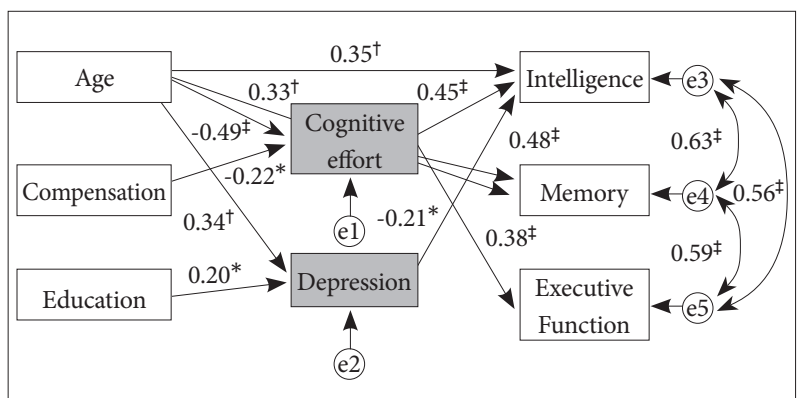

Figure 1. Final path model for cognitive function in patients with mild traumatic brain injury. ${ }^{*} p<0.05,{ }^{\dagger} p<0.01,{ }^{\ddagger} p<0.001$.

intelligence scores. Whereas age had positive direct influences on intelligence and memory, it had negative indirect effects on cognitive functions, through cognitive effort. Consequently, the total effect of age on intelligence $(\beta=0.05, p=0.577)$ and memory $(\beta=0.05, p=0.598)$ was small and not significant. The total effect of age on executive functions was negative ( $\beta=-0.22$, $\mathrm{p}<0.001)$. Workers' compensation had only an indirect effect on cognitive functions, through cognitive effort. The total effects of workers' compensation on each examined cognitive function were significant and negative: intelligence ( $\beta=-0.10$, $\mathrm{p}=0.034)$, memory $(\beta=-0.11, \mathrm{p}=0.032)$, and executive functions ( $\beta=-0.08, p=0.038)$. The total effect of education on intelligence was not significant $(\beta=-0.04, p=0.139)$. Gender and abnormal imaging findings had no direct or indirect effects on cognitive functions. Contrary to our a priori hypothesis, depression had no causative role in cognitive effort.

\section{DISCUSSION}

The primary purpose of this study was to examine the potential mediating effects of depression and cognitive effort on cognitive functions in patients with mTBI. Our results demonstrate that only cognitive effort had significant mediating ef- 
fects on intelligence, memory, and executive functions. Depression had no causative relationship with cognitive effort, whereas workers' compensation did have a significant negative effect on cognitive effort, although the magnitude was modest. These results suggest that cognitive functioning in mTBI seems to be significantly influenced by external factors such as workers' compensation and insufficient effort.

As Rey's 15-Item Test is a type of cognitive test, one might argue that the positive relationship between this memory test and other cognitive tests is inevitable. However, as described in the Methods section, the level of difficulty of Rey's 15-Item Test is extremely low. A previous study demonstrated that even patients with severe TBI could attain a perfect score (15). ${ }^{26}$ Hence, in general, Rey 15-Item Test scores should show a strong negative skew, reflecting a ceiling effect. However, the distribution of scores in our study was not skewed, and only 36 of 115 (31.3\%) patients had perfect scores. Thus, the results suggest that a substantial proportion of our participants did not do their best on the cognitive test.

It is noteworthy that depression had no causative influence on cognitive effort. These results suggest that the degree to which patients with mTBI exerted effort would be more plausibly attributed to external factors, such as compensation, rather than to intrinsic factors, such as depression. Additionally, most cognitive function test results are more strongly associated with cognitive effort than with severity of depression, which also supports the undue influence of external factors on the cognitive performance in patients with mTBI. On the other hand, the mean severity of depression in our sample was above $60 \mathrm{~T}$, which is higher than normal. Depression is a prevalent psychiatric symptom in mTBI, possibly due to neural impairments, including catecholamine dysregulation ${ }^{27}$ and neuroanatomical changes. ${ }^{28}$ Although depression was not significantly associated with cognitive functions or efforts, as was compensation, careful evaluation and management are needed to prevent the development of depressive disorders.

Another interesting finding in our study was the relationship between workers' compensation and cognitive effort. Workers' compensation had indirect negative effects on intelligence, memory, and executive functions, through insufficient cognitive effort. This is consistent with findings that malingering or intentional cognitive underperformance were most common in workers' compensation cases $(30.12 \%)$, followed by personal injury cases (28.66\%), criminal cases (19.25\%), and medical or psychiatric cases $(8.11 \%)$, categorized according to referral type. ${ }^{29}$ Specifically, these patterns of exaggeration or cognitive underperformance were considered the most pronounced in patients with mild head injury. Although several previous mTBI studies have regarded patients seeking compensation as one group, ${ }^{30,31}$ we examined differences between those receiving workers' compensation and others as we thought that workers' compensation would be more strongly related to secondary gain. Medical care costs and disability benefits might be similar between those receiving workers' compensation and others, but workers' compensation also covered injury-related offwork costs. For example, in Korea, temporary disability benefits corresponding to $70 \%$ of average daily wages are paid to a worker with work-related injuries for off-work days (4 days or longer) during treatment. ${ }^{32}$

Finally, age had positive direct effects and negative indirect effects on intelligence and memory, which consequently led to non-significant total effects. Age only had indirect effects on executive functions. Executive functioning was the only cognitive domain exclusively mediated by cognitive effort. As executive functions organize various cognitive functions to perform goal-directed activities, it has been suggested that cognitive effort is closely related to executive functions in mTBI. ${ }^{33}$ Generally, age is associated with worse psychosocial and cognitive functioning. Several studies have reported that patients with traumatic brain injury had worse psychosocial and cognitive functioning as a function of age. ${ }^{34,35}$ Those findings seem contrary to our findings suggesting that intelligence and memory functions were positively associated with ageing. The discrepancy might arise from the use of different measures. Whereas previous studies measured the outcomes several years to decades after injury, the mean post-injury period of our sample was about 10 months. Another possible reason is individual differences in the relationship between ageing and cognitive functions. Recent studies have suggested that individual differences in genetic profiles influence intelligence across the lifespan. $^{36}$

There are several limitations to this study. First, we could not compare neuropsychological tests between compensation and non-compensation groups. We would need to assess patients with mTBI who are not seeking compensation for their injuries to more accurately evaluate the role of compensation. However, in Korea, 92.5\% of adults over 20 years old are covered by one or more types of private insurance, ${ }^{37}$ so there are very few patients for whom compensation is irrelevant. Additionally, because most of the patients in this study were referred for neuropsychological tests, we could not confirm their medical status at the initial period of injury. In the same manner as in several previous studies, ${ }^{31}$ identifying ratings on the Glasgow coma scale was difficult due to lack of information from the ambulance service or the emergency department. To minimize selection bias, we comprehensively reviewed the records and included only those patients who were strongly suggested to have $\mathrm{mTBI}$ based on the definition recommended by the CDC. ${ }^{12}$ Finally, the relatively large proportion (52/115, $45.2 \%$ ) of abnormal MRI findings in our sample should be 
noted. This proportion of abnormal imaging findings is higher than that reported in previous studies, where it ranged from $16 \%$ to $32 \%{ }^{38,39}$ We speculate that this is due to the characteristics of our sample. As our subjects were referred to our hospital for comprehensive cognitive testing, complicated cases were more frequent than it was in other samples. The high prevalence of abnormal imaging findings was not associated with psychiatric symptoms or cognitive domains, which is consistent with previous studies. ${ }^{40}$

\section{Conclusions}

In summary, this study demonstrates that insufficient cognitive effort is a strong mediating factor for various cognitive functions in mTBI, including intelligence, memory, and executive functions. Additionally, patients receiving workers' compensation showed significantly less cognitive effort than others. Our results revealed that clinicians who evaluate and manage patients with mTBI often feel that those patients might not do their best in tests of cognitive functions. We believe that our results will be helpful in clinical practice with regard to evaluating and interpreting the results obtained by patients with mTBI on cognitive tests. Health professionals should encourage patients with mTBI to perform cognitive tests with sufficient effort.

\section{Acknowledgments} Fund.

This work was supported by the Soonchunhyang University Research

\section{REFERENCES}

1. Faul M, Xu L, Wald MM, Coronado VG. Traumatic Brain Injury in the United States: Emergency Department Visits, Hospitalizations and Deaths 2002-2006. In: Control NCfIPa, Editor. Centers for Disease Control and Prevention. Atlanta, GA: National Center for Injury Prevention and Control, Centers for Disease Control and Prevention; 2010.

2. Niess C, Grauel U, Toennes SW, Bratzke H. Incidence of axonal injury in human brain tissue. Acta Neuropathol 2002;104:79-84.

3. Kushner D. Mild traumatic brain injury: toward understanding manifestations and treatment. Arch Intern Med 1998;158:1617-1624.

4. Alexander MP. Minor traumatic brain injury: a review of physiogenesis and psychogenesis. Semin Clin Neuropsychiatry 1997;2:177-187.

5. Cassidy JD, Carroll LJ, Peloso PM, Borg J, von Holst H, Holm L, et al. Incidence, risk factors and prevention of mild traumatic brain injury: results of the WHO Collaborating Centre Task Force on Mild Traumatic Brain Injury. J Rehabil Med 2004;(43 Suppl):28-60.

6. Williams WH, Potter S, Ryland H. Mild traumatic brain injury and Postconcussion Syndrome: a neuropsychological perspective. J Neurol Neurosurg Psychiatry 2010;81:1116-1122.

7. Schretlen DJ, Shapiro AM. A quantitative review of the effects of traumatic brain injury on cognitive functioning. Int Rev Psychiatry 2003;15: 341-349.

8. Sigurdardottir S, Andelic N, Roe C, Jerstad T, Schanke AK. Post-concussion symptoms after traumatic brain injury at 3 and 12 months post-injury: a prospective study. Brain Inj 2009;23:489-497.

9. Flaro L, Green P, Robertson E. Word Memory Test failure 23 times higher in mild brain injury than in parents seeking custody: the power of external incentives. Brain Inj 2007;21:373-383.

10. Jamora CW, Young A, Ruff RM. Comparison of subjective cognitive complaints with neuropsychological tests in individuals with mild vs more severe traumatic brain injuries. Brain Inj 2012;26:36-47.

11. Lange RT, Iverson GL, Rose A. Depression strongly influences postconcussion symptom reporting following mild traumatic brain injury. J Head Trauma Rehabil 2011;26:127-137.

12. National Center for Injury Prevention and Control. Report to Congress on Mild Traumatic Brain Injury in the United States: Steps to Prevent a Serious Public Health Problem. Atlanta, GA: Centers for Disease Control and Prevention; 2003.

13. Rey A. The Clinical Examination in Psychology. Paris: University Press of France; 1964.

14. Slick DJ, Tan JE, Strauss EH, Hultsch DF. Detecting malingering: a survey of experts' practices. Arch Clin Neuropsychol 2004;19:465-473.

15. Reznek L. The Rey 15-item memory test for malingering: a meta-analysis. Brain Inj 2005;19:539-543.

16. Oh SW. Review of the Korean Wechsler Adult Intelligence Scale-Revised. Wonkwang Psychiatry 1995;11:27-47.

17. Kim HK. Assessment of Memory Disorders Using Rey-Kim Memory Test. Korean J Rehab Psychol 2001;8:29-48.

18. Perret E. The left frontal lobe of man and the suppression of habitual responses in verbal categorical behaviour. Neuropsychologia 1974;12: 323-330.

19. Kim HK. Clinical evaluation of the Frontal Lobe Syndrome using Kims Frontal-Executive Neuropsychological Test. Korean J Rehab Psychol 2001;8:173-190.

20. Benton AL, Hamsher K. Multilingual Aphasia Examination Manual. Iowa: University of Iowa; 1989.

21. Berning LC, Weed NC, Aloia MS. Interrater reliability of the Ruff Figural Fluency Test. Assessment 1998;5:181-186.

22. Derogatis LR, Lipman RS, Covi L. SCL-90: an outpatient psychiatric rating scale--preliminary report. Psychopharmacol Bull 1973;9:13-28.

23. Derogatis LR, Rickels K, Rock AF. The SCL-90 and the MMPI: a step in the validation of a new self-report scale. Br J Psychiatry 1976;128: 280-289.

24. Rauter UK, Leonard CE, Swett CP. SCL-90-R factor structure in an acute, involuntary, adult psychiatric inpatient sample. J Clin Psychol 1996;52:625-629.

25. Hoofien D, Barak O, Vakil E, Gilboa A. Symptom checklist-90 revised scores in persons with traumatic brain injury: affective reactions or neurobehavioral outcomes of the injury? Appl Neuropsychol 2005;12: 30-39.

26. Taylor LA, Kreutzer JS, West DD. Evaluation of malingering cut-off scores for the Rey 15-Item Test: a brain injury case study series. Brain Inj 2003; 17:295-308.

27. McAllister TW, Flashman LA, McDonald BC, Saykin AJ. Mechanisms of working memory dysfunction after mild and moderate TBI: evidence from functional MRI and neurogenetics. J Neurotrauma 2006; 23:1450-1467.

28. Stulemeijer M, Vos PE, van der Werf S, van Dijk G, Rijpkema M, Fernández G. How mild traumatic brain injury may affect declarative memory performance in the post-acute stage. J Neurotrauma 2010;27:1585-1595.

29. Mittenberg W, Patton C, Canyock EM, Condit DC. Base rates of malingering and symptom exaggeration. J Clin Exp Neuropsychol 2002; 24:1094-1102.

30. Schiehser DM, Delis DC, Filoteo JV, Delano-Wood L, Han SD, Jak AJ, et al. Are self-reported symptoms of executive dysfunction associated with objective executive function performance following mild to moderate traumatic brain injury? J Clin Exp Neuropsychol 2011;33:704-714.

31. Armistead-Jehle P. Symptom validity test performance in U.S. veterans referred for evaluation of mild TBI. Appl Neuropsychol 2010;17:52-59.

32. Korea Workers' Compensation \& Welfare Service. Practical manual for unemployment insurance and workers' compensation. Seoul: Ko- 
rea Worker's Compensation \& Welface Service; 2010

33. Ord JS, Greve KW, Bianchini KJ, Aguerrevere LE. Executive dysfunction in traumatic brain injury: the effects of injury severity and effort on the Wisconsin Card Sorting Test. J Clin Exp Neuropsychol 2010; 32:132-140.

34. Kolakowsky-Hayner SA, Hammond FM, Wright J, Novack TA, Englander J, Diaz-Arrastia R, et al. Ageing and traumatic brain injury: age, decline in function and level of assistance over the first 10 years postinjury. Brain Inj 2012;26:1328-1337.

35. Senathi-Raja D, Ponsford J, Schonberger M. Impact of age on longterm cognitive function after traumatic brain injury. Neuropsychology 2010;24:336-344.

36. Deary IJ, Yang J, Davies G, Harris SE, Tenesa A, Liewald D, et al. Ge- netic contributions to stability and change in intelligence from childhood to old age. Nature 2012;482:212-215.

37. Kim DH, Choi W. 2011 Insurance Consumer Survey. Seoul: Korea Insurance Research Institute; 2011

38. Hughes DG, Jackson A, Mason DL, Berry E, Hollis S, Yates DW. Abnormalities on magnetic resonance imaging seen acutely following mild traumatic brain injury: correlation with neuropsychological tests and delayed recovery. Neuroradiology 2004;46:550-558.

39. Iverson GL, Lovell MR, Smith S, Franzen MD. Prevalence of abnormal CT-scans following mild head injury. Brain Inj 2000;14:1057-1061.

40. Lee H, Wintermark M, Gean AD, Ghajar J, Manley GT, Mukherjee P. Focal lesions in acute mild traumatic brain injury and neurocognitive outcome: CT versus 3T MRI. J Neurotrauma 2008;25:1049-1056. 